\title{
Abnormal amino acid metabolism in patients with early stage Alzheimer dementia
}

\author{
D. Fekkes ${ }^{1}$, T. J. M. van der Cammen², C. P. M. van Loon'2, C. Verschoor ${ }^{2}$, \\ F. van Harskamp ${ }^{3}$, I. de Koning ${ }^{3}$, W. J. Schudel ${ }^{4}$, and \\ L. Pepplinkhuizen ${ }^{1}$
}

\begin{abstract}
${ }^{1}$ Department of Psychiatry, Section Pathophysiology of Behaviour, ${ }^{2}$ Department of Internal Medicine I and Geriatric Medicine, ${ }^{3}$ Department of Neurology, and ${ }^{4}$ Department of Psychiatry, Erasmus University Rotterdam, The Netherlands
\end{abstract}

Accepted February 2, 1998;

received November 11, 1997

Summary. Plasma levels of several amino acids were studied in 14 patients with early stage probable Alzheimer's disease (AD) and 17 age-matched controls. In the AD patients a possible relationship between amino acid levels and behavioural symptomatology was also investigated. We found significantly reduced levels of tryptophan and methionine in plasma samples from the AD patients compared to the control subjects. Moreover, plasma tyrosine/ large neutral amino acids (LNAA) ratio and the ratio of plasma taurine and the product of the plasma levels of methionine and serine (TSM-ratio) were significantly increased in the AD patients in comparison with the controls. However, no difference was found in plasma tryptophan/LNAA ratio and in homocysteine levels between both groups. Concerning the behavioural symptomatology no significant correlation was found between the Reisberg Behave AD scale and plasma amino acid levels or ratios. The reported findings suggest that abnormal amino acid metabolism is present in the early stages of AD. We hypothesize that this abnormality could play a role in the pathogenesis of behavioural changes occurring in later stages of AD.

Keywords: Alzheimer's disease, amino acids, behavioural pathology, transmethylation, tryptophan, methionine.

\section{Introduction}

The vitamins B12 and folic acid are important coenzymes involved in the onecarbon metabolism, which generates S-adenosyl-methionine (SAM). Both folate and vitamin B12 deficiency may cause neurological and psychiatric disturbances including dementia (Bottiglieri et al., 1994). The methylB12 dependent enzyme methionine synthetase catalyzes the transfer of a 
methyl group from 5-methyltetrahydrofolate to homocysteine, generating tetrahydrofolate and methionine. Homocysteine is produced entirely from the methylation cycle, as it is totally absent from any dietary source. Trolin et al. (1995) have found a decreased methionine adenosyltransferase activity in erythrocytes of patients with dementia disorders. In the same study, borderline high levels of serum homocysteine were detected. Nilsson et al. (1996) found increased plasma homocysteine concentrations in both demented and non-demented persons with psychiatric problems compared to control subjects (i.e., persons without cognitive and psychiatric problems).

In some studies, the authors determined the concentrations of free amino acids in plasma of patients suffering from dementia. Two studies (Basun et al., 1990; Rudman et al., 1989) found a lower concentration of tryptophan in Alzheimer's Disease (AD) patients. Basun et al. (1990) found a mean concentration $( \pm \mathrm{SD})$ of $30 \pm 15 \mu \mathrm{mol} / \mathrm{l}$ in $\mathrm{AD}$ patients and $41 \pm 18 \mu \mathrm{mol} / \mathrm{l}$ in controls. They found no difference in the concentration of methionine. In the other study (Rudman et al., 1989) the mean concentrations ( \pm SEM) of tryptophan were $44.3 \pm 1.6 \mu \mathrm{mol} / 1$ and $54.9 \pm 2.4 \mu \mathrm{mol} / 1$, respectively. These investigators also found a lower methionine concentration in AD patients $(24.1 \pm 0.6 \mu \mathrm{mol} /$ 1) than in their control group $(28.6 \pm 0.7 \mu \mathrm{mol} / \mathrm{l})$. In one study (Martinez et al., 1993) no difference in tryptophan levels was found between Alzheimer patients and healthy aged persons $(34 \pm 10 \mu \mathrm{mol} / 1$ and $35 \pm 13 \mu \mathrm{mol} / \mathrm{l}$, respectively). These investigators suggest that an abnormal amino acid metabolism may play a role in the symptomatology of AD.

Because the results concerning the amino acid status in AD are somewhat contradictory, we decided to study the amino acid metabolism in AD in relation with behavioural pathology. Next to the free plasma amino acids, we also determined some amino acid ratios. These comprised the ratios of tryptophan and tyrosine to the sum of the other large neutral amino acids (LNAA: tyrosine/tryptophan, phenylalanine, valine, leucine and isoleucine), which compete for the transport of the respective amino acid through the bloodbrain barrier. These ratios reflect the amount of tryptophan and tyrosine available for serotonin and noradrenaline/dopamine synthesis in the central nervous system, respectively (Fernström and Wurtman, 1972). A reduced serotonin synthesis would explain some of the behavioural disturbances (anxiety, depression, agitation, sleeplessness) seen in AD. Besides, we determined the so-called TSM-ratio, which is defined as the ratio of 100 times the taurine concentration in plasma and the product of the plasma concentrations of serine and methionine. This ratio is a reflection of the status of the amino acids involved in transmethylation processes (Fekkes et al., 1994; Fekkes and Pepplinkhuizen, 1997).

\section{Methods}

\section{Subjects}

Fourteen patients were derived from the Memory Clinic of the University Hospital Rotterdam Dijkzigt. The patients' characteristics are shown in Table 1. Informed consent was obtained from all patients and their main representative. All patients underwent a full comprehensive geriatric assessment (Rubenstein et al., 1984; Epstein et al., 1987) includ- 
Table 1. Study group characteristics

\begin{tabular}{lll}
\hline Characteristics & $\begin{array}{l}\text { AD patients } \\
(\mathrm{n}=14)\end{array}$ & $\begin{array}{l}\text { Controls } \\
(\mathrm{n}=17)\end{array}$ \\
\hline Mean age ( \pm SD; years; range) & $\begin{array}{l}73.6 \pm 6.3 \\
(65-82)\end{array}$ & $\begin{array}{l}70.1 \pm 1.3 \\
(68-73)\end{array}$ \\
$\begin{array}{l}\text { Gender (M/F) } \\
\text { Median GDS total (range) }\end{array}$ & $4.5(3-6)$ & $17 / 0$ \\
Median CDR total (range) $^{\mathrm{b}}$ & $1(0.5-3)$ & \\
$\begin{array}{l}\text { Median Reisberg Behave AD } \\
\text { Global score (range) }\end{array}$ & $1(0-2)$ & \\
$\begin{array}{l}\text { Median Reisberg Behave AD } \\
\text { Total score (range) }\end{array}$ & $0.5(0-5)$ & \\
\hline
\end{tabular}

a The Global Deterioration Scale (GDS) has a range of 17 points. ${ }^{\mathrm{b}}$ The Clinical Dementia Rating Scale (CDR) has a range of $0.5-5.0$ points. ${ }^{\mathrm{c}}$ The global score of the Reisberg Behave AD scale has a range of $0-3$ points. ${ }^{\mathrm{d}}$ The total score of the Reisberg Behave AD scale has a range of 0-75 points

ing history and informant history, medication history, and physical, neurological and neuropsychological examination. Exclusion criteria were the following: psychiatric and organic mental disorders other than dementia, alcohol abuse, and use of neuroleptics and anticonvulsive agents.

For all patients, a consensus diagnosis of dementia was obtained by the Memory Clinic team consisting of two geriatricians, a neurologist, a neuropsychologist and a psychiatrist. The diagnosis of dementia was verified according to a standard protocol including instrumental evaluation by computer tomography. Dementia was diagnosed according to DSM-III-R criteria (American Psychiatric Association, 1987). The subdiagnosis of probable AD was based on the criteria of the working group of the NINCDSADRDA (McKhann et al., 1984). Severity of dementia was staged according to the Global Deterioration Scale (GDS) (Reisberg et al., 1982) and the Clinical Dementia Rating Scale (CDR) (Hughes et al., 1982).

The GDS is a scale of seven points, with a score of 3 indicating mild memory problems with independent functioning, a score of 4 indicating mild memory problems with a reduced capacity in some aspects of daily functioning, a score of 5 indicating moderate cognitive problems and dependence on others in daily life, and a score of 6 indicating severe cognitive problems and total dependence on others in daily life. The CDR varies from 0.5 to 5.0 points, with a score of 0.5 indicating questionnable dementia, a score of 1 indicating mild dementia, a score of 2 indicating moderate dementia, and a score of 3 indicating severe dementia.

Depressive and behavioural symptomatology was obtained from the history with the patient and the informant recorded by a psychiatric interview and a score on the Reisberg Behave AD Scale (Reisberg et al., 1987), a scale which measures behavioural changes in $\mathrm{AD}$. The total score of this scale varies from 0 to 75 points, and the global score varies from 0 to 3 points. The global score measures the burden on the caregiver due to the behavioural changes in $\mathrm{AD}$. All patients underwent extensive laboratory investigations, including vitamin $B_{12}$ and folate levels. All had normal folate and vitamin $B_{12}$ levels (reference interval: $120-640 \mathrm{pmol} / \mathrm{l} \mathrm{serum}$ ) at the time of blood sampling.

The controls consisted of 17 healthy elderly men with normal cognition (based on interviews taken by specially trained health professionals and the mini mental state examination) and without a diagnosis of any psychiatric or neurologic disease (Table 1). 


\section{Blood sampling and biochemical analyses}

Blood ( $4 \mathrm{ml})$ was drawn between 11.00 and 12.00 a.m. from the anterocubital vein using $\mathrm{K}_{3}$-EDTA Vacutainer tubes. Immediately after sampling the blood was centrifuged for $20 \mathrm{~min}$ at $2650 \mathrm{~g}\left(20^{\circ} \mathrm{C}\right)$ and the plasma was frozen at $-80^{\circ} \mathrm{C}$ until analysis. The plasma amino acids were analyzed by high-performance liquid chromatography (HPLC) using pre-column derivatization with o-phthaldialdehyde (Fekkes et al., 1995). The tryptophanratio was calculated by dividing the total tryptophan level by the sum of the other LNAA, which compete for the transport of tryptophan through the blood-brain barrier. The tyrosine-ratio was calculated in the same manner by substituting tryptophan for tyrosine. The TSM-ratio was calculated by dividing 100 times the taurine concentration in plasma by the product of the plasma concentrations of serine and methionine.

Total homocysteine in plasma was measured by HPLC with fluorescence detection using a minor modification of the method described by Araki and Sako (1987). Separation was carried out at $28^{\circ} \mathrm{C}$ with a Spherisorb ODS 2 column $(150 \times 3.0 \mathrm{~mm}$ I.D., $5 \mu \mathrm{m}$ particle size) from Shandon, protected by an ODS Hypersil guard column $(20 \times 2.1 \mathrm{~mm}$ I.D. $)$ from Hewlett Packard. A binary gradient from $0.15 \mathrm{M}$ sodium phosphate buffer ( $\mathrm{pH} 1.75)$ to acetonitril $(0-95 \%)$ at a flow-rate of $0.6 \mathrm{ml} / \mathrm{min}$ was applied.

\section{Statistical analysis}

All values are given as mean \pm SD. Data were analyzed using a Mann Whitney U Wilcoxon test for statistical significance. In a further analysis we calculated the sum of the items of the Behave AD-Scale according to Reisberg. We used the Pearson correlation coefficient to find a correlation between behavioural changes and the amino acid levels in plasma.

A logistic regression analysis showed no difference between men and women. Moreover, the mean levels of the biochemical parameters were equal between men and women.

\section{Results}

We found that the concentration of the amino acid tryptophan in the plasma of the patients with early stage probable AD was significantly lower than in the plasma of the controls (viz. $33.9 \pm 7.0$ and $44.1 \pm 5.4 \mu \mathrm{mol} / 1$, respectively; $\mathrm{p}=0.0004$; Table 2). These lower tryptophan levels in the AD patients may have implications for the synthesis of the neurotransmitter serotonin for which this amino acid is the precursor. However, the ratio of plasma tryptophan to the sum of the other LNAA was not different between the two groups (AD: $7.98 \pm 1.67$; controls: $7.85 \pm 1.15$ ). The ratio of plasma tyrosine to the sum of the LNAA in the AD patients $(14.64 \pm 1.99)$ was significantly higher than the ratio in the control group $(10.77 \pm 1.38 ; \mathrm{p}<0.0001)$. This latter amino acid is the precursor of both the neurotransmitters dopamine and noradrenaline.

The plasma levels of the amino acid methionine, the direct precursor of the principal methylgroup donor SAM, were also significantly lower in the AD patients $(18.2 \pm 3.5 \mu \mathrm{mol} / \mathrm{l})$ than in the controls $(24.94 \pm 2.75 \mu \mathrm{mol} / \mathrm{l} ; \mathrm{p}<$ $0.0001)$. Moreover, the ratio of plasma taurine and the product of the plasma levels of methionine and serine (TSM-ratio), which is a measure of deranged transmethylation, was much higher in the AD patients than in the control group $(3.11 \pm 1.23$ vs. $1.72 \pm 0.49$, respectively; $p<0.0001)$. These results suggest that aberrations in the transmethylation reactions are present in $\mathrm{AD}$ 
Table 2. Concentrations $(\mu \mathrm{mol} / \mathrm{L}$, mean $\pm \mathrm{SD})$ and ratios $($ mean $\pm \mathrm{SD})$ of amino acids in plasma

\begin{tabular}{lcc}
\hline Amino acid/ratio & $\begin{array}{c}\text { AD patients } \\
(\mathrm{n}=14)\end{array}$ & $\begin{array}{l}\text { Controls } \\
(\mathrm{n}=17)\end{array}$ \\
\hline Tryptophan & $33.9 \pm 7.0^{\mathrm{a}}$ & $44.1 \pm 5.4$ \\
Methionine & $18.2 \pm 3.5^{\mathrm{b}}$ & $24.9 \pm 2.8$ \\
Homocysteine & $19.4 \pm 9.2$ & $17.9 \pm 3.5$ \\
Tryptophan/LNAA* & $7.98 \pm 1.67$ & $7.85 \pm 1.15$ \\
Tyrosine/LNAA* & $14.64 \pm 1.99^{\mathrm{b}}$ & $10.77 \pm 1.38$ \\
TSM** $^{* *}$ & $3.11 \pm 1.23^{\mathrm{b}}$ & $1.72 \pm 0.49$ \\
\hline
\end{tabular}

Amino acid levels were determined as described in Methods. * $L N A A$ Large neutral amino acids, **TSM ratio of plasma taurine and the product of the plasma levels of methionine and serine. ${ }^{\mathrm{a}} \mathrm{p}=0.0004,{ }^{\mathrm{b}} \mathrm{p}<0.0001$ as compared to controls

patients. On the other hand, the plasma concentrations of homocysteine in AD patients were not different from those in the control group (19.4 \pm 9.2 and $17.9 \pm 3.5 \mu \mathrm{mol} / 1$, respectively; Table 2).

We found no significant correlation between behavioural changes and the amino acid levels. However, there was a slight correlation between the TSMratio and the Reisberg Behave AD scale $(r=-0.48 ; \mathrm{p}=0.08)$.

\section{Discussion}

Our results concerning the lower tryptophan concentration in the plasma of AD patients compared to a control group are in agreement with those reported in other studies (Basun et al., 1990; Rudman et al., 1989; Shaw et al., 1981; Watkins et al., 1989). Although Martinez et al. (1993) found no difference between the plasma levels of tryptophan in AD patients and controls, their reported reference values were much lower than those found by other investigators.

The lower tryptophan concentrations in the plasma of AD patients may result in a reduced synthesis of serotonin. On the other hand, our results on the tryptophan/LNAA ratio suggest than the central serotonin synthesis is not limited in these patients. We also failed to demonstrate a significant correlation between amino acid levels and behavioural changes in our AD patients. However, our patient group was small and most patients presented to the Memory Clinic rather early in the course of their dementia with very little behavioural symptomatology. One could wonder whether the disturbances in the amino acid metabolism in our AD patients could be a pathogenetic factor for behavioural symptomatology which usually occurs in the later stages of AD.

Our finding that the tryptophan/LNAA ratio in AD patients is not different from the ratio in the controls - suggesting no deficit in the central 
serotonergic activity - is in line with the low depressive and behavioural symptomatology in these patients. On the other hand, we found the tyrosine/ LNAA ratio to be increased in the plasma of $\mathrm{AD}$ patients resulting in a possibly higher central dopaminergic and/or noradrenergic activity. However, there was no correlation with agitated behaviour.

With regard to transmethylation mechanisms, there have been reports on a consistent accumulation of homocysteine and its transsulfuration product cystathionine in the serum of vitamin $\mathrm{B}_{12}$ deficient and $\mathrm{AD}$ patients. It has been suggested (Bell et al., 1992) that the amino acid homocysteine, which is both a vascular disease risk factor and a precursor of the excitotoxic amino acids cysteine and homocysteic acid, could play a role in the pathophysiology of dementia in older patients and in depressive symptomatology in AD. Homocysteine was found highest in older depressed patients with concomitant vascular disease and correlated negatively with folate and vitamin $\mathrm{B}_{12}$ levels (Bell et al., 1992; Carmel et al., 1995). On the other hand, higher homocysteine correlated significantly with poorer cognition only in the nonvascular depressed group (Bell et al., 1992). Lower concentrations of vitamin $B_{12}$ and folate and higher concentrations of homocysteine have also been associated with poorer spatial copying skills (Riggs et al., 1996). However, our AD patients showed normal mean plasma homocysteine levels. These normal homocysteine concentrations may be the result of the normal levels of vitamin $B_{12}$ and folate found in our patients.

Next to the above mentioned studies on homocysteine and vitamin $B_{12}$, some other studies on the one-carbon metabolism in AD patients are known. Morrison et al. (1996) found decreased levels of SAM in some brain areas compared with matched controls. Gomes-Trolin et al. (1995) reported on a decreased activity of the enzyme methionine adenosyltransferase (MAT) in erythrocytes of AD patients and an increased MAT affinity towards methionine. Treatment with $\mathrm{B}_{12}, \mathrm{SAM}$ and folate caused a significant decrease in homocysteine in parallel with a significant decrease in affinity for MAT. This group also reported on an increased MAT activity in the cortex gyrus frontalis of AD patients irrespective of the serum $\mathrm{B}_{12}$ levels (Gomes-Trolin et al., 1996).

We and other investigators (Rudman et al., 1989) found lowed concentrations of methionine - the substrate for the above mentioned enzyme MAT and the precursor of the active methyl donor SAM - in the plasma of AD patients. Methyl transfer is among others involved in the degradation of noradrenaline and the synthesis of choline. A decrease of the latter cholinergic marker in the CSF of AD patients has also been reported (Kumar and Giacobini, 1988). These findings support the hypothesis that aberrations in transmethylation reactions might be involved in the pathogenesis of AD. This is also supported by the finding that the so-called TSM-ratio, which is thought to be a marker of the status of the amino acids involved in transmethylation processes, is higher in AD than in age-matched controls. It has to be remarked that the impaired transmethylation found in this study is not caused by a low $\mathrm{B} 12$ or folic acid state of the AD patients as the levels of these vitamins were normal. This latter finding on vitamin $\mathrm{B}_{12}$ is in agreement with the observation 
of Gomes-Trolin et al. (1996), who found increased MAT activity in brain samples from $A D$ patients with normal as well as low levels of serum $B_{12}$.

In conclusion, this study suggests that both deviant concentrations of some plasma amino acids and abnormal transmethylation reactions already occur in the early stages of AD. Our preliminary results indicate a possible imbalance between serotonergic and dopaminergic/noradrenergic neurotransmission processes in AD patients. Since we only investigated 14 early stage AD patients, we cannot be conclusive about the clinical effects of the abnormalities found in the amino acid metabolism. Longitudinal studies in larger samples are required in order to establish a possible link with the symptomatology of AD.

\section{Acknowledgements}

We thank the patients for participation in this study, and A. van Dalen-van Duyne and A. C. C. Voskuilen-Kooyman for their technical assistance.

\section{References}

American Psychiatric Association (1987) Diagnostic and statistical manual of mental disorders, 3rd ed (revised). American Psychiatric Association, Washington $\mathrm{DC}$

Araki A, Sako Y (1987) Determination of free and total homocysteine in human plasma by high-performance liquid chromatography with fluorescence detection. J Chrom 422: 43-52

Basun H, Forssell LG, Almkvist O, Cowburn RF, Eklof R, Winblad B, Wetterberg L (1990) Amino acid concentrations in cerebrospinal fluid and plasma in Alzheimer's disease and healthy control subjects. J Neural Transm [PD Sect] 2: 295-304

Bell IR, Edman JS, Selhub J, Morrow FD, Marby DW, Kayne HL, Cole JO (1992) Plasma homocysteine in vascular disease and in nonvascular dementia of depressed elderly people. Acta Psychiatr Scand 86: 386-390

Bottiglieri T, Hyland K, Reynolds EH (1994) The clinical potential of ademetionine (Sadenosylmethionine) in neurological disorders (Review). Drugs 48: 137-152

Carmel R, Gott PS, Waters CH, Cairo K, Green R, Bondareff W, DeGiorgio CM, Cummings JL, Jacobsen DW, Buckwalter G, Henderson VW (1995) The frequently low cobalamin levels in dementia usually signify treatable metabolic, neurologic and electrophysiologic abnormalities. Eur J Haematol 54: 245-253

Epstein AM, Hall JA, Besdine R, Cumella BA Jr., Feldstein M, McNeil BJ, Rowe JW (1987) The emergence of geriatric assessment units: the "new technology of geriatrics". Ann Intern Med 106: 299-303

Fekkes D, Pepplinkhuizen L (1997) Amino acid studies in transient acute polymorphic psychosis. Amino Acids 12: 107-117

Fekkes D, Pepplinkhuizen L, Verheij R, Bruinvels J (1994) Abnormal plasma levels of serine, methionine, and taurine in transient acute polymorphic psychosis. Psychiatry Res 51: 11-18

Fekkes D, van Dalen A, Edelman M, Voskuilen A (1995) Validation of the determination of amino acids in plasma by high-performance liquid chromatography using automated derivatization with o-phthaldialdehyde. J Chromatogr B: Biomed Appl 669: $177-186$

Fernström JD, Wurtman RJ (1972) Brain serotonin content: physiological regulation by plasma neutral amino acids. Science 178: 414-416

Gomes-Trolin C, Regland B, Oreland L (1995) Decreased methionine adenosyltransferase activity in erythrocytes of patients with dementia disorders. Eur Neuropsychopharmacol 5: 107-114 
Gomes-Trolin C, Gottfries CG, Regland B, Oreland L (1996) Influence of vitamin B B $_{12}$ on brain methionine adenosyltransferase activity in senile dementia of the Alzheimer's type. J Neural Transm [Gen Sect] 103: 861-872

Hughes CP, Berg L, Danziger WL, Coben LA, Martin RL (1982) A new clinical scale for the staging of dementia. Br J Psychiatry 140: 566-572

Kumar V, Giacobini E (1988) Cerebrospinal fluid choline, and acetylcholinesterase activity in familial vs. non-familial Alzheimer's disease patients. Arch Gerontol Geriatr 7: $11-17$

Martinez M, Frank A, Diez-Tejedor E, Hernanz A (1993) Amino acid concentrations in cerebrospinal fluid and serum in Alzheimer's disease and vascular dementia. J Neural Transm [PD Sect] 6: 1-9

McKhann G, Drachman D, Folstein M, Katzman R, Price D, Stadlan EM (1984) Clinical diagnosis of Alzheimer disease: report of the NINCDS-ADRDA Working Group under the auspices of the Department of Health and Human Services Task Force on Alzheimer Disease. Neurology 34:939-944

Morrison LD, Smith DD, Kish SJ (1996) Brain S-adenosylmethionine levels are severely decreased in Alzheimer's disease. J Neurochem 67: 1328-1331

Nilsson K, Gustafson L, Fäldt R, Andersson A, Brattström L, Lindgren A, Israelsson B, Hultberg B (1996) Hyperhomocysteinaemia - a common finding in a psychogeriatric population. Eur J Clin Invest 26: 853-859

Reisberg B, Ferris SH, De Leon MJ, Crook T (1982) The Global Deterioration Scale for assessment of primary degenerative dementia. Am J Psychiatry 139: 1136-1139

Reisberg B, Borenstein J. Franssen E, Salob S, Steinberg G, Shulman E, Ferris SH, Georgotas A (1987) BEHAVE-AD: a clinical rating scale for the assessment of pharmacologically remediable behavioral symptomatology in Alzheimer's disease: problems, prospects, and perspectives. J Clin Psychiatry 48 [Suppl] 5: 1-16

Riggs KM, Spiro III A, Tucker K, Rush D (1996) Relations of vitamin B-12, vitamin B6 , folate, and homocysteine to cognitive performance in the Normative Aging Study. Am J Clin Nutr 63: 306-314

Rubenstein LZ, Josephson KR, Wieland GD, English PA, Sayre JA, Kane RL (1984) Effectiveness of a geriatric evaluation unit: a randomized clinical trial. NEJM 311: $1664-1670$

Rudman D, Mattson DE, Feller AG, Cotter R, Johnson RC (1989) Fasting plasma amino acids in elderly men. Am J Clin Nutr 49: 559-566

Shaw DM, Tidmarsh SF, Karajgi BM, Sweeney EA, Williams S, Elameer M, Twining C (1981) Pilot study of amino acids in senile dementia (Letter). Br J Psychiatry 139: 580-582

Watkins SE, Thomas DE, Clifford M, Tidmarsh SF, Sweeney AE, Ah-Sing E, Dickerson JW, Cowie VA, Shaw DW (1989) Plasma amino acids in patients with senile dementia and in subjects with Down's syndrome at an age vulnerable to Alzheimer changes. J Ment Defic Res 33: 159-166

Authors' address: Dr. D. Fekkes, Department of Psychiatry, Section Pathophysiology of Behaviour, Erasmus University Rotterdam, P.O. Box 1738, 3000 DR Rotterdam, The Netherlands. 